\title{
TWO PROBLEMS IN SINGULAR PERTURBATIONS OF DIFFERENTIAL EQUATIONS
}

\author{
K. W. CHANG \\ (Received 23 October 1967, revised 11 December 1967)
}

\section{Introduction}

Let $\varepsilon>0$ be a small real parameter, let $y, z$ be real $m$-dimensional and $n$-dimensional vectors respectively and let $f, g$ be respectively real $m$ dimensional and $n$-dimensional vector functions of their arguments. This paper aims to discuss the following two problems in singular perturbations.

(A) Consider first the autonomous system

$$
\begin{aligned}
y^{\prime} & =f(y, z, \varepsilon), \\
\varepsilon z^{\prime} & =g(y, z, \varepsilon) .
\end{aligned}
$$

$$
\left({ }^{\prime}=d / d t\right)
$$

Here under the assumption that the degenerate system obtained from (1) by setting $\varepsilon=0$ has a periodic solution with period $T$ in $t$, one looks for suitable conditions which will ensure that (1) also has a periodic solution with a period which tends to $T$ as $\varepsilon \rightarrow 0$. One also discusses the stability of the closed path of this periodic solution as $\varepsilon \rightarrow 0$. Friedrichs and Wasow [6] were the first to discuss a singular perturbation problem of this nature. Recently Anosov [1] has also treated this problem (1) and to the best of our knowledge his results are the most general which have been obtained. His method of approach is based on the notion of the so-called rapid motion system associated with (1).

Our purpose here is to establish Anosov's results [1; Theorems 3, 4] by an entirely different and shorter method. Our method makes direct use of the concept of orthonormal system of coordinates (cf. Urabe [13]). More important, we find that we can improve on Anosov's Theorem 5 regarding the behaviour, as $\varepsilon \rightarrow 0$, of solutions of (1) which are close to the unique closed path (see Theorem 4 in the last section).

(B) Consider next the following non-autonomous system

$$
\begin{aligned}
y^{\prime} & =f(y, z, t, \varepsilon), \\
\varepsilon z^{\prime} & =g(y, z, t, \varepsilon),
\end{aligned}
$$

where $t$ varies over the entire half-line $t \geqq 0$. Here again one looks for suitable assumptions which will ensure that (2) possesses a solution bounded 
for all $t \geqq 0$ in some neighbourhood of a bounded solution of the degenerate system of (2). Another aspect of the problem is to study the behaviour of this solution as $\varepsilon \rightarrow 0$. This problem has already been treated by Butuzov [2] and Hoppensteadt [8]. Their results are extensions of the corresponding results of Levin [10] and Tihonov [12] who had earlier dealt with the initial value problem with $t$ restricted to a finite $t$-interval. We notice however an important difference between the results of Tihonov-Hoppensteadt and Levin-Butunov: the former considered the 'stable' case, whereas the latter allowed conditional stability. (Note also that Tihonov's result has been shown to be false by Hoppensteadt [9] who has also shown how it can be corrected.)

We have succeeded (Theorem 2 below) in extending Butuzov's Theorem 1 in two directions. Firstly, his assumption 4, which had earlier appeared as $\mathrm{H3}$ in Levin's paper cited above, will be replaced by a more natural assumption, namely, assumption (IV) of section 3. Secondly, instead of his assumption 3, which implies that a certain linear equation, the equation (9) below, has uniform asymptotic stability, we simply require that it possesses an exponential dichotomy.

We also investigate the behaviour as $\varepsilon \rightarrow 0$ of every solution of (2) and our results here (Theorem 3 ) we believe to be new.

The plan of this paper is as follows. In the next section we prove Theorem 1 which embodies Anosov's Theorems 3, 4. In section 3 we prove Theorem 2. In section 4 we state and prove a lemma from which Theorem 3 will later be deduced in section $\mathbf{5}$. In the last section Theorem 4 is obtained by an application of Theorem 3 .

\section{Statement and Proof of Theorem 1}

In this section and section 6 we assume that the functions $f, g$ of (1) together with the Jacobian matrices $f_{y}, f_{z}, g_{y}, g_{z}$ are continuous in their arguments $(y, z, \varepsilon)$. Order symbols such as $0(\varepsilon), o(1)$ refer to $\varepsilon \rightarrow 0$ and for any continuous function $x(t)$ we write $\|x(t)\|=\sup _{-\infty<t<\infty}|x(t)|$.

THEOREM 1. Let the following assumptions hold:

(I) The equation $g(y, z, 0)=0$ has a continuously differentiable solution $z=p(y)$ defined for $y \in D$ where $D$ is some region in $y$-space.

(II) The equation

$$
y^{\prime}=f[y, \varphi(y), 0]=\bar{f}(y)
$$

possesses in $D$ a nonconstant periodic solution $u(t)$ with period $T$ in $t$.

(III) The variational equation of (3)

$$
v^{\prime}=f_{y}[u(t)] v
$$


admits a single multiplier equal to one (or, equivalently, has $(m-1)$ characteristic exponents $\neq 0$ ).

(IV) Every eigenvalue of $g_{z}[u(t), \varphi[u(t)], 0]$ has real part different from zero.

Then for $\varepsilon$ sufficiently small, the system (1) has a periodic solution $y_{\varepsilon}(t), z_{\varepsilon}(t)^{1}$ with period $T+o(1)$ in $t$ such that, as $\varepsilon \rightarrow 0$, the closed path of this solution tends to the closed path of the periodic solution $u(t), \varphi[u(t)]$ of the degenerate system.

Furthermore there exists $\rho>0$ such that (1) has no other periodic solution (apart from translations in $t$ ) whose path remains in the $\rho$-neighbourhood of $u(t), \varphi[u(t)]$ and whose period differs from $T$ by less than $\rho$.

Proof. It follows from assumption (II) that $\bar{f}[u(t)] \neq 0$ is a periodic function with period $T$ in $t$. As is known (Halanay [7]) there exists a change of coordinates

$$
y=u(\theta)+S(\theta) x
$$

where $\theta$ is a scalar, $x$ an $(m-1)$ vector and $S(\theta)$ an $m \times(m-1)$ matrix function with period $T$ in $\theta$ whose columns are mutually orthogonal. By this change of coordinates the solution $y=u(t)$ is now defined by $x=0$, $\theta=t$. Also the first equation of $(1)$ is transformed into

$$
\bar{f}[u(\theta)] \theta^{\prime}+\theta^{\prime}(d S(\theta) / d \theta) x+S(\theta) x^{\prime}=f[u(\theta)+S(\theta) x, z, \varepsilon]
$$

As in Halanay [7; p. 280], by forming the scalar product of this equation with the columns of $S(\theta)$ and with an $m^{\text {th }}$ vector orthogonal to these columns, we obtain respectively

$$
\begin{aligned}
& x^{\prime}=X(x, z, \theta, \varepsilon), \\
& \theta^{\prime}=\Theta(x, z, \theta, \varepsilon),
\end{aligned}
$$

where $\Theta, X$ are periodic functions in $\theta$ with period $T$, are continuous in $(x, z, \theta, \varepsilon)$ and such that

$$
\begin{aligned}
\Theta[0, \varphi[u(\theta)], \theta, 0] & \equiv \mathbf{1}, \\
X[0, \varphi[u(\theta)], \theta, 0] & \equiv 0 .
\end{aligned}
$$

Thus there exists $\varepsilon_{0}>0$ such that $\Theta \neq 0$ for $|x|,|z-\varphi[u(\theta)]|, \varepsilon \leqq \varepsilon_{0}$. We can therefore take $\theta$ to be the new independent variable and express (1) in the form

$$
\begin{aligned}
\frac{d x}{d \theta} & =\frac{X(x, z, \theta, \varepsilon)}{\Theta(x, z, \theta, \varepsilon)} \equiv F(x, z, \theta, \varepsilon), \\
\varepsilon \frac{d z}{d \theta} & =\frac{g[u(\theta)+S(\theta) x, z, \theta, \varepsilon]}{\Theta(x, z, \theta, \varepsilon)} \equiv G(x, z, \theta, \varepsilon) .
\end{aligned}
$$

1 The subscript here and elsewhere is used simply to denote dependence on $\varepsilon$, not partial differentiation with respect to $\varepsilon$. 
For $\varepsilon=0$ the system (6) has the solution $x=0, z=\varphi[u(\theta)]$. We shall show that (6) has a unique periodic solution with period $T$ in $\theta$ by verifying that all the hypotheses (I), (II'), (III') in Chang [3] are satisfied.

First we have

$$
\begin{aligned}
B(\theta) & =G_{z}(\theta)=\left[g_{z}(\theta) \Theta-g \Theta_{z}\right] /\left.\Theta^{2}\right|_{x=0, \varepsilon=0, z=\varphi[u(\theta)]} \\
& =g_{z}[u(\theta), \varphi[u(\theta)], 0],
\end{aligned}
$$

since $\Theta=1$ and $g=0$ for $\varepsilon=0, x=0, z=\varphi[u(\theta)]$. Therefore by (IV) the real part of every eigenvalue of $B(\theta)$ has absolute value $\geqq \mu$, for some $\mu>0$ independent of $\theta$.

Similarly we obtain

$$
\begin{aligned}
A_{1}(\theta) & =F_{x}(\theta)-F_{z}(\theta) G_{z}^{-1}(\theta) G_{x}(\theta) \\
& =X_{x}(\theta)-X_{z}(\theta) g_{z}^{-1}(\theta) g_{y}(\theta) S(\theta) .
\end{aligned}
$$

Now under the transformation (5) the equation (3) becomes

$$
\frac{d x}{d \theta}=\frac{X[x, \varphi[u(\theta)+S(\theta) x], \theta, 0]}{\Theta[x, \varphi[u(\theta)+S(\theta) x], \theta, 0]} .
$$

The variational equation of (7) has the coefficient matrix $C(\theta)$ obtained by taking the derivative of the right hand side of (7) with respect to $x$ at the point $x=0$. It is easily verified that in fact

$$
C(\theta)=A_{1}(\theta) \text {. }
$$

Moreover $($ see $[7 ;$ p. 285]) the $(m \rightarrow 1)$ characteristic exponents of the linear periodic equation

$$
\frac{d v}{d \theta}=C(\theta) v
$$

are identical with the $m$ characteristic exponents of the equation (4) with 0 omitted. Therefore by hypothesis (III) these characteristic exponents are different from zero, and so the equation $d v / d \theta=C(\theta) v=A_{1}(\theta) v$ has no nontrivial periodic solution with period $T$ in $\theta$.

Thus (6) satisfies all the hypotheses in [3] and it follows that there exists $\rho_{0}>0$ such that for all $\varepsilon$ sufficiently small the system (6) possesses a unique periodic solution $x_{\varepsilon}(\theta), z_{\varepsilon}(\theta)$ with period $T$ in $\theta$ such that

Moreover

$$
\left\|x_{\varepsilon}(\theta)\right\|+\left\|z_{\varepsilon}(\theta)-\varphi[u(\theta)]\right\| \leqq \rho_{0} .
$$

$$
\left\|x_{\varepsilon}(\theta)\right\|+\left\|z_{\varepsilon}(\theta)-\varphi[u(\theta)]\right\| \rightarrow 0 \text { as } \varepsilon \rightarrow 0 .
$$

To this unique periodic solution corresponds a solution

$$
\begin{aligned}
& \tilde{y}_{\varepsilon}(t)=u[\theta(t)]+S[\theta(t)] x_{\varepsilon}[\theta(t)] \\
& \tilde{z}_{\varepsilon}(t)=z_{\varepsilon}[\theta(t)]
\end{aligned}
$$


of the system (1), where $\theta(t)$ is obtained by inverting the relation

$$
t-t_{0}=\int_{0}^{\theta} \frac{d \theta}{\Theta\left[x_{\varepsilon}(\theta), z_{\varepsilon}(\theta), \theta, \varepsilon\right]} .
$$

An increase in $\theta$ by $T$ corresponds to an increase in $t$ by

$$
\widetilde{T}=\int_{0}^{T} \frac{d \theta}{\Theta\left[x_{\varepsilon}(\theta), z_{\varepsilon}(\theta), \theta, \varepsilon\right]}=T+o(1),
$$

since $\Theta\left[x_{\varepsilon}(\theta), z_{\varepsilon}(\theta), \theta, \varepsilon\right]=1+0\left(\left|x_{\varepsilon}\right|+\left|z_{\varepsilon}\right|+\varepsilon\right)=1+o(1)$.

It follows that the solution $\tilde{y}_{\varepsilon}(t), \tilde{z}_{\varepsilon}(t)$ of $(1)$ is periodic in $t$ with period $\tilde{T}$. Moreover the closed path of $\tilde{y}_{\varepsilon}(t), \tilde{z}_{\varepsilon}(t)$ tends to the closed path of $u(t)$, $\varphi[u(t)]$ as $\varepsilon \rightarrow 0$.

We now prove the last statement of Theorem 1. Assume that (1) has a periodic solution $y(t), z(t)$ whose path lies in the $\rho$-neighbourhood of $u(t)$, $\varphi[u(t)]$ and whose period is $\tilde{T}=T+\tilde{\rho}$ where $|\tilde{\rho}| \leqq \rho$. We first show that $\theta$ increases by $T$ when $t$ increases by $\tilde{T}$. In fact, since $\theta^{\prime}$ is positive and close to 1 , it follows that

$$
\theta(t+\tilde{T})-\theta(t) \text { is close to } \tilde{T} .
$$

On the other hand, since $\theta$ is determined uniquely $(\bmod T)$ by $y$, it follows from $y(t+\tilde{T})=y(t)$ that

$$
\theta(t+\widetilde{T})-\theta(t) \text { is a multiple of } T \text {, say } k T \text {. }
$$

Since $T$ is close to $T, k$ must be 1 .

Next it follows from $y(t)=u[\theta(t)]+S[\theta(t)] x(t)$ that $x(t+\widetilde{T})=x(t)$ and therefore, as a function of $\theta, x$ has period $T$. Similarly, since $z(t+\tilde{T})=z(t)$ it follows that as a function of $\theta, z$ also has period $T$. By what has been proved earlier the solution $x, z$ of $(6)$ with period $T$ in $\theta$ coincides with the solution $x_{\varepsilon}(\theta), z_{\varepsilon}(\theta)$ already found. Hence, for some real $t_{0}, y(t)=\tilde{y}_{\varepsilon}\left(t+t_{0}\right), z(t)=\tilde{z}_{\varepsilon}\left(t+t_{0}\right)$ and this completes the proof.

\section{Theorem 2 and its Proof}

In order to state our results we make the following assumptions. In what follows, $f_{y}(t)$ denotes the Jacobian matrix $f_{y}[\bar{y}(t), \bar{z}(t), t, 0]$ and similar meanings are attached to $f_{z}(t), g_{y}(t), g_{z}(t), g_{t}(t)$.

(I) The degenerate system

$$
\begin{aligned}
y^{\prime} & =f(y, z, t, 0), \\
0 & =g(y, z, t, 0),
\end{aligned}
$$

has a continuous bounded solution $\bar{y}(t), \bar{z}(t)$ for $0 \leqq t<\infty$. 
(II) The function $f$ and the Jacobian matrices $f_{y}, f_{z}, f_{t}$ are continuous and bounded in $(y, z, t, \varepsilon)$ for $|y-\bar{y}(t)|,|z-\bar{z}(t)|, \varepsilon \leqq N$ and $0 \leqq t<\infty$. Also

$$
\|f[\bar{y}(t), \bar{z}(t), t, \varepsilon]-f[\bar{y}(t), \bar{z}(t), t, 0]\| \rightarrow 0 \text { as } \varepsilon \rightarrow 0,
$$

where $\|f\|$ now denotes $\sup _{t \geq 0}|f(t)|$, and to any $\delta>0$ there exists $\omega=\omega(\delta)$, $0<\omega<\delta$ such that for $|v|,|w|, \varepsilon \leqq \omega$

$$
\left\|f_{y}[\bar{y}(t)+v, \bar{z}(t)+w-U(t) v, t, \varepsilon]-f_{y}[\bar{y}(t), \bar{z}(t), t, 0]\right\| \leqq \delta
$$

where $U(t)=g_{z}^{-1}(t) g_{y}(t)$ and similarly for $f_{z}$. The function $g$ also satisfies similar assumptions.

(III) There exists a constant $\mu_{0}>0$ such that the real part of every eigenvalue of $g_{z}(t)$ has absolute value $\geqq \mu_{0}$.

It follows that

$$
\left|\operatorname{det} g_{z}(t)\right| \geqq \mu_{0}^{n} \text { for } t \geqq 0 .
$$

Hence $g_{z}^{-1}(t)$ exists, is continuous and bounded and so is $U(t)=g_{z}^{-1}(t) g_{y}(t)$. By $(8 \mathrm{a}) \bar{y}^{\prime}(t)$ exists, is continuous and bounded. By $(8 \mathrm{~b})$ and the implicit function theorem $\bar{z}^{\prime}(t)$ also exists, in fact,

$$
\bar{z}^{\prime}(t)=-g_{z}^{-1}(t)\left[g_{y}(t) \bar{y}^{\prime}(t)+g_{t}(t)\right] .
$$

It follows that if $g$ has continuous and bounded second derivatives in $t, y, z$ for $|y-\bar{y}(t)|,|z-\bar{z}(t)|, \varepsilon \leqq N$ and $t \geqq 0$, then $U^{\prime}(t)$ will exist and be continuous and bounded. Instead of assuming the existence of second derivatives of $g$ we shall simply assume

(IV) $U(t)=g_{z}^{-1}(t) g_{y}(t)$ has a continuous and bounded derivative on $0 \leqq t<\infty$.

(V) The linear equation

$$
v^{\prime}=\left[f_{y}(t)-f_{z}(t) U(t)\right] v
$$

possesses an exponential dichotomy, that is, (9) has a fundamental matrix $V(t), V(0)=I_{m}$, such that

$$
\begin{aligned}
\left|V(t) P V^{-1}(s)\right| \leqq K e^{-\nu(t-s)} & \text { for } \quad t \geqq s, \\
\left|V(t)\left(I_{m}-P\right) V^{-1}(s)\right| \leqq K e^{-\nu(8-t)} & \text { for } \quad s \geqq t,
\end{aligned}
$$

where $P$ is a projection matrix, $I_{m}$ the $m \times m$ unit matrix and $K, v$ are positive constants.

It follows from (III) that the matrix $g_{z}(t)$ has the same number $k$ of eigenvalues with negative real parts for every $t$. We now recall lemmas $1,2,3$ of [3] by means of which we have established that the linear equation

$$
\varepsilon w^{\prime}=g_{z}(t) w=B(t) w
$$

possesses an exponential dichotomy, in fact, for all sufficiently small $\varepsilon>0$, 
(11) has a fundamental matrix $W(t), W(0)=I_{n}$, satisfying

$$
\begin{aligned}
& \left|W(t) P_{1} W^{-1}(s)\right| \leqq L e^{-\mu(t-s) / \varepsilon} \quad \text { for } t \geqq s, \\
& \left|W(t)\left(I_{n}-P_{1}\right) W^{-1}(s)\right| \leqq L e^{-\mu(s-t) / \varepsilon} \text { for } s \geqq t \text {, }
\end{aligned}
$$

where $\mu=\mu_{0} / 8, P_{1}$ is a projection matrix of rank $k, I_{n}$ the $n \times n$ unit matrix and $L$ is some positive constant independent of $\varepsilon$.

Let

$$
v=y-\bar{y}(t), w=z-\bar{z}(t)+U(t) v
$$

then we have

THEOREM 2. Let the above assumptions (I)-(V) hold. Then given $\rho>0$ there exist positive constants $\gamma, \varepsilon_{0}$ such that for any vectors $v_{0}, w_{0}$ with $v_{0}=P v_{0}, w_{0}=P_{1} w_{0},\left|v_{0}\right| \leqq \gamma,\left|w_{0}\right| \leqq \gamma$ and for $0<\varepsilon \leqq \varepsilon_{0}$, the system (2) has a unique solution $y_{\varepsilon}(t), z_{\varepsilon}(t)$ throughout the interval $0 \leqq t<\infty$ which satisfies

$$
\begin{aligned}
P\left[y_{\varepsilon}(0)-\bar{y}(0)\right] & =v_{0}, \\
P_{1}\left[z_{\varepsilon}(0)-\bar{z}(0)+U(0)\left\{y_{\varepsilon}(0)-\bar{y}(0)\right\}\right] & =w_{0}
\end{aligned}
$$

and

$$
\left\|y_{\varepsilon}(t)-\bar{y}(t)\right\|+\left\|z_{\varepsilon}(t)-\bar{z}(t)\right\| \leqq \rho .
$$

The proof of Theorem 2 is based on the following lemma $A$.

LEMмa A. Suppose that the linear equation

$$
y^{\prime}=A(t) y
$$

has a fundamental matrix $Y(t)$ with $Y(0)=I$ such that

$$
\begin{aligned}
& \left|Y(t) P Y^{-1}(s)\right| \leqq H e^{-\alpha(t-s)} \quad \text { for } \quad t \geqq s, \\
& \left|Y(t)(I-P) Y^{-1}(s)\right| \leqq H e^{-\alpha(s-t)} \quad \text { for } \quad s \geqq t \text {, }
\end{aligned}
$$

where $P$ and $I-P$ are supplementary projections and $H, \alpha$ are positive constants. Let $b(t)$ be a continuous bounded function of $t$. Then for any vector $x_{0}=P x_{0}$ the equation

$$
x^{\prime}=A(t) x+b(t)
$$

has a unique bounded solution $x(t)$ such that $P x(0)=x_{0}$. Moreover

$$
\|x(t)\| \leqq H\left|x_{0}\right|+C\|b(t)\|,
$$

where $C=2 H \alpha^{-1}$ is a positive constant depending only on $H, \alpha$.

Proof. The function

$$
x(t)=Y(t) x_{0}+\int_{0}^{t} Y(t) P Y^{-1}(s) b(s) d s-\int_{t}^{\infty} Y(t)(I-P) Y^{-1}(s) b(s) d s
$$


exists for all $t \geqq 0$ and it is seen by differentiation that $x(t)$ is indeed a solution of (17) with the required properties. It is the only bounded solution of (17) with $P x(0)=x_{0}$, since the homogeneous equation has no nontrivial bounded solution with $P x(0)=0$.

Proof of Theorem 2. As in [3] the change of variables (13) takes (2) into the system

$$
\begin{aligned}
v^{\prime} & =A_{1}(t) v+A_{2}(t) w+F(v, w, t, \varepsilon), \\
\varepsilon w^{\prime} & =B(t) w+G(v, w, t, \varepsilon)
\end{aligned}
$$

where

$$
A_{1}(t)=f_{y}(t)-f_{z}(t) U(t) ; \quad A_{2}(t)=f_{z}(t) ; \quad B(t)=g_{z}(t) .
$$

The functions $F, G$ are continuous in $(v, w, t, \varepsilon)$ such that

$$
\|F(0,0, t, \varepsilon)\|+\|G(0,0, t, \varepsilon)\| \rightarrow 0 \text { as } \varepsilon \rightarrow 0 .
$$

Moreover to any $\delta>0$ there is a corresponding $\omega=\omega(\delta), 0<\omega<\delta$, such that

$$
\begin{aligned}
& |F(v, w, t, \varepsilon)-F(\tilde{v}, \tilde{w}, t, \varepsilon)| \leqq \delta(|v-\tilde{v}|+|w-\tilde{w}|), \\
& |G(v, w, t, \varepsilon)-G(\tilde{v}, \tilde{w}, t, \varepsilon)| \leqq \delta(|v-\tilde{v}|+|w-\tilde{w}|),
\end{aligned}
$$

for all $t \geqq 0$ if $|v|,|\tilde{v}|,|w|,|\tilde{w}|, \varepsilon \leqq \omega$.

For any pair of continuous bounded functions $\xi(t), \eta(t)$ consider the system

$$
\begin{aligned}
v^{\prime} & =A_{1}(t) v+A_{2}(t) w+F[\xi(t), \eta(t), t, \varepsilon], \\
\varepsilon w^{\prime} & =B(t) w+G[\xi(t), \eta(t), t, \varepsilon] .
\end{aligned}
$$

We show first that $(19 \mathrm{~b})$ has a unique solution $w(t)$ which will then be substituted into (19a). Since (11) has a fundamental matrix satisfying (12) it follows by lemma $\mathrm{A}$ that for any vector $w_{0}$ with $w_{0}=P_{1} w_{0}$ the equation $(19 \mathrm{~b})$ has a unique bounded solution $\rightsquigarrow(t)$ such that

and

$$
P_{1} w(0)=w_{0}
$$

$$
\begin{aligned}
\|w(t)\| & \leqq L\left|w_{0}\right|+C_{1}|| G(\xi(t), \eta(t), t, \varepsilon) \| \\
& \leqq L\left|w_{0}\right|+C_{1}[\|G(0,0, t, \varepsilon)\|+\delta\|\xi(t)\|+\delta\|\eta(t)\|],
\end{aligned}
$$

if $\|\xi(t)\|,\|\eta(t)\|, \varepsilon \leqq \omega$, where $C_{1}=C_{1}(L, \mu)$ is a constant. By lemma A again for any vector $v_{0}$ with $v_{0}=P v_{0}$ the equation

$$
v^{\prime}=A_{1}(t) v+A_{2}(t) w(t)+F[\xi(t), \eta(t), t, \varepsilon],
$$

has a unique bounded solution $v(t)$ such that

and

$$
P v(0)=v_{0}
$$




$$
\|v(t)\| \leqq K\left|v_{0}\right|+C_{2}\left[\left\|A_{2}(t)\right\|\|\mid w(t)\|+\|F(0,0, t, \varepsilon)\|+\delta\|\xi(t)\|+\delta\|\eta(t)\|\right],
$$

where $C_{2}=C_{2}(K, v)$.

Next choose $\left|v_{0}\right|,\left|w_{0}\right|$ so small that

$$
K\left|v_{0}\right|+L\left[1+C_{2}|| A_{2}(t)||\right]\left|w_{0}\right| \leqq \frac{1}{4} \omega,
$$

and choose $\delta$ so small that

$$
\delta\left(C_{1}+C_{2}+C_{1} C_{2}\left\|A_{2}(t)\right\|\right) \leqq \frac{1}{2},
$$

and then choose $\varepsilon_{0} \leqq \omega$ so small that

$$
C_{2}\|F(0,0, t, \varepsilon)\|+\left(C_{1}+C_{1} C_{2}\left\|A_{2}(t)\right\|\right)\|G(0,0, t, \varepsilon)\| \leqq \frac{1}{4} \omega
$$

for $0<\varepsilon \leqq \varepsilon_{0}$. Then if $0<\varepsilon \leqq \varepsilon_{0}$ we obtain

$$
\begin{aligned}
\|v(t)\|+\|w(t)\| & \leqq \frac{1}{4} \omega+\frac{1}{4} \omega+\frac{1}{2}(\|\xi(t)\|+\|\eta(t)\|) \\
& =\frac{1}{2} \omega+\frac{1}{2}(\|\xi(t)\|+\|\eta(t)\|) .
\end{aligned}
$$

The set of all pairs of bounded continuous functions $(\xi, \eta)$ is a Banach space if we define

$$
\begin{aligned}
(\xi, \eta)+(\xi, \tilde{\eta}) & =(\xi+\xi, \eta+\tilde{\eta}) \\
\beta(\xi, \eta) & =(\beta \xi, \beta \eta) \\
\|(\xi, \eta)\| & =\|\xi(t)\|+\|\eta(t)\| .
\end{aligned}
$$

Denote by $S$ the set consisting of all pairs $(\xi, \eta)$ with $\|(\xi, \eta)\| \leqq \omega$. Then the mapping $(\xi, \eta) \rightarrow(v, w)$ maps $S$ into itself. We show next that this mapping is a contraction. Suppose then $\left(\xi_{1}, \eta_{1}\right)$ and $\left(\xi_{2}, \eta_{2}\right)$ are pairs in $S$ and $\left(v_{1}, w_{1}\right)$ and $\left(v_{2}, w_{2}\right)$ are the corresponding images. Then

$$
\psi(t)=v_{1}(t)-v_{2}(t) \text { and } \zeta(t)=w_{1}(t)-w_{2}(t)
$$

are the solutions of

$$
\begin{aligned}
\psi^{\prime} & =A_{1}(t) \psi+A_{2}(t) \zeta+F\left[\xi_{1}(t), \eta_{1}(t), t, \varepsilon\right]-F\left[\xi_{2}(t), \eta_{2}(t), t, \varepsilon\right] \\
\varepsilon \zeta^{\prime} & =B(t) \zeta+G\left[\xi_{1}(t), \eta_{1}(t), t, \varepsilon\right]-G\left[\xi_{2}(t), \eta_{2}(t), t, \varepsilon\right] .
\end{aligned}
$$

It follows, as above, that

$$
\begin{aligned}
& \|\zeta(t)\| \leqq C_{1} \delta\left[\left\|\xi_{1}(t)-\xi_{2}(t)\right\|+\left\|\eta_{1}(t)-\eta_{2}(t)\right\|\right], \\
& \|\psi(t)\| \leqq C_{2} \delta\left[\left\|\xi_{1}(t)-\xi_{2}(t)\right\|+\left\|\eta_{1}(t)-\eta_{2}(t)\right\|\right]+C_{2}\left\|A_{2}(t)\right\|\|\zeta(t)\| .
\end{aligned}
$$

Hence

$$
\left\|v_{1}(t)-v_{2}(t)\right\|+\left\|w_{1}(t)-w_{2}(t)\right\| \leqq \frac{1}{2}\left[\left\|\xi_{1}(t)-\xi_{2}(t)\right\|+\left\|\eta_{1}(t)-\eta_{2}(t)\right\|\right] .
$$

It follows from the principle of contraction mappings that $S$ has a unique fixed point. Therefore, for any vectors $v_{0}, w_{0}$ such that $v_{0}=P v_{0}, w_{0}=P_{1} w_{0}$ and (20) holds and for $\varepsilon$ sufficiently small, the system (18) has a unique 
bounded solution $v(t)$, $w(t)$ satisfying (14) and $\|v(t)\|+\|w(t)\| \leqq \omega$. Hence given $\rho>0$ there exist positive constants $\gamma, \varepsilon_{0}$ such that for any vectors $v_{0}, w_{0}$ with $v_{0}=P v_{0}, w_{0}=P_{1} w_{0}$ and $\left|v_{0}\right|,\left|w_{0}\right| \leqq \gamma$ and for $0<\varepsilon \leqq \varepsilon_{0}$, the original system (2) has a unique solution $y_{\varepsilon}(t), z_{\varepsilon}(t)$ which satisfies (14) and (15). This completes the proof of Theorem 2.

\section{4}

In this section we prove the following lemma which may be of interest by itself. It is an extension of lemma 7 of Coppel [5].

Lemma B. Suppose that $v_{1}(t), v_{2}(t), w_{1}(t), w_{2}(t)$ are continuous vector functions such that for $t \geqq \tau \geqq t_{0}$,

$$
\begin{aligned}
\left|v_{1}(t)\right| & \leqq K e^{-\nu(t-\tau)}\left|v_{1}(\tau)\right|+K \gamma \int_{\tau}^{t} e^{-\nu(t-s)}(|v(s)|+|w(s)|) d s, \\
\left|v_{2}(\tau)\right| & \leqq K e^{-\nu(t-\tau)}\left|v_{2}(t)\right|+K \gamma \int_{\tau}^{t} e^{-\nu(s-\tau)}(|v(s)|+|w(s)|) d s, \\
\left|w_{1}(t)\right| & \leqq L e^{-\mu(t-\tau) / \varepsilon}\left|w_{1}(\tau)\right|+L \gamma \varepsilon^{-1} \int_{\tau}^{t} e^{-\mu(t-s) / \varepsilon}(|v(s)|+|w(s)|) d s, \\
\left|w_{2}(\tau)\right| & \leqq L e^{-\mu(t-\tau) / \varepsilon}\left|w_{2}(t)\right|+L \gamma \varepsilon^{-1} \int_{\tau}^{t} e^{-\mu(s-\tau) / \varepsilon}(|v(s)|+|w(s)|) d s,
\end{aligned}
$$

where $K, L, \gamma, \nu, \mu, \varepsilon$ are positive constants and $v=v_{1}+v_{2}, w=w_{1}+w_{2}$.

Then if $\gamma, \varepsilon$ are sufficiently small, we have either (i)

$$
\left|v_{1}(t)\right|+\left|w_{1}(t)\right| \leqq(K+L) e^{-\lambda(t-\tau)}\left(\left|v_{1}(\tau)\right|+\left|w_{1}(\tau)\right|\right) \quad \text { for } t \geqq \tau \geqq t_{0},
$$

and

$$
\left|v_{2}(t)\right|+\left|w_{2}(t)\right| \leqq\left|v_{1}(t)\right|+\left|w_{1}(t)\right| \quad \text { for } t \geqq t_{0},
$$

or (ii) for some $t_{1} \geqq t_{0}$,

$$
\left|v_{2}(\tau)\right|+\left|w_{2}(\tau)\right| \leqq(K+L) e^{-\lambda_{1}(t-\tau)}\left(\left|v_{2}(t)\right|+\left|w_{2}(t)\right|\right) \quad \text { for } t \geqq \tau \geqq t_{1},
$$

and

$$
\left|v_{1}(t)\right|+\left|w_{1}(t)\right| \leqq \eta\left(\left|v_{2}(t)\right|+\left|w_{2}(t)\right|\right) \quad \text { for } t \geqq t_{1},
$$

where $\lambda, \lambda_{1}$ and $\eta>1$ are positive constants depending only on $K, L, \nu$.

Proof. We can assume without loss of generality that $K \geqq 1, L \geqq 1$. Put $\eta=\mathbf{l}+(K+L)^{2}$. We shall show that lemma $\mathrm{B}$ holds if we choose $\gamma>0$ so small that

$$
\gamma(1+\eta)(K+L)^{2}\left(\mu^{-1}+v^{-1}\right)<1
$$

and then choose $\varepsilon>0$ so small that

In fact it follows that

$$
\varepsilon<2 \gamma \nu^{-1} K \text {. }
$$




$$
\varepsilon<1 \text { and } 2 \gamma(1+\eta)(K+L)<\frac{\mu \nu}{\mu+\nu}<\mu, \nu .
$$

A fortiori,

$$
\mathbf{4} \gamma(K+L)<\mu, v
$$

Moreover

$$
\begin{aligned}
\delta_{1} & =\mu-\gamma L(1+\eta)-\varepsilon[\nu-\gamma K(1+\eta)] \\
& >\frac{1}{2} \mu+\gamma K(1+\eta)-\varepsilon \nu \\
& >\frac{1}{2} \mu \\
& >\gamma(K+L)(1+\eta)
\end{aligned}
$$

and hence

$$
\begin{aligned}
\lambda_{1} & =\nu-\gamma K(1+\eta)-\gamma^{2} K L(1+\eta)^{2} \delta_{1}^{-1} \\
& >\nu-\gamma K(1+\eta)-\gamma L(1+\eta) \\
& >\frac{1}{2} \nu .
\end{aligned}
$$

Similarly

$$
\begin{gathered}
\delta=\mu-2 \gamma L-\varepsilon(\nu-2 \gamma K) \\
>\frac{1}{2} \mu>2 \gamma(K+L)
\end{gathered}
$$

and

$$
\begin{aligned}
\lambda & =\nu-2 \gamma K-4 \gamma^{2} K L \delta^{-1} \\
& >\frac{1}{2} \nu .
\end{aligned}
$$

Suppose first that the solution $v_{i}(t), w_{i}(t), i=1,2$ of the integral inequalities (21) has the property that

$$
\left|v_{1}(t)\right|+\left|w_{1}(t)\right| \geqq\left|v_{2}(t)\right|+\left|w_{2}(t)\right| \text { for all } t \geqq t_{0} .
$$

Then for $t \geqq \tau \geqq t_{0}$ by the first and third inequalities of $(21)$

$$
\begin{aligned}
& \left|v_{1}(t)\right| \leqq K e^{-\nu(t-\tau)}\left|v_{1}(\tau)\right|+2 K \gamma \int_{\tau}^{t} e^{-\nu(t-s)}\left(\left|v_{1}(s)\right|+\left|w_{1}(s)\right|\right) d s, \\
& \left|w_{1}(t)\right| \leqq L e^{-\mu(t-\tau) / \varepsilon}\left|w_{1}(\tau)\right|+2 L \gamma \varepsilon^{-1} \int_{\tau}^{t} e^{-\mu(t-s) / \varepsilon}\left(\left|v_{1}(s)\right|+\left|w_{1}(s)\right|\right) d s .
\end{aligned}
$$

Since $v>2 K \gamma$ and $\mu>2 L \gamma$ it follows by Gronwall's lemma [4; p. 19] that (22a) $\left|v_{1}(t)\right| \leqq K e^{-(\nu-2 K \gamma)(t-\tau)}\left|v_{1}(\tau)\right|+2 K \gamma \int_{\tau}^{t} e^{-(\nu-2 K \gamma)(t-8)}\left|w_{1}(s)\right| d s$, (22b) $\left|w_{1}(t)\right| \leqq L e^{-(\mu-2 L \gamma)(t-\tau) / \varepsilon}\left|w_{1}(\tau)\right|+2 L \gamma \varepsilon^{-1} \int_{\tau}^{t} e^{-(\mu-2 L \gamma)(t-s) / \varepsilon}\left|v_{1}(s)\right| d s$.

On substituting for $\left|w_{1}(s)\right|$ in (22a) by means of (22b) we get

where

$$
\left|v_{1}(t)\right| \leqq K e^{-(\nu-2 K \gamma)(t-\tau)}\left|v_{1}(\tau)\right|+I_{1}+I_{2},
$$

$$
I_{1}=2 K \gamma \cdot L\left|w_{1}(\tau)\right| \int_{\tau}^{t} e^{-(\nu-2 K \gamma)(t-s)} e^{-(\mu-2 L \gamma)(s-\tau) / \varepsilon} d s
$$

and

$$
I_{2}=2 K \gamma \cdot 2 L \gamma \varepsilon^{-1} \int_{\tau}^{t} e^{-(\nu-2 K \gamma)(t-s)}\left\{\int_{\tau}^{s} e^{-(\mu-2 L \gamma)(s-\theta) / \varepsilon}\left|v_{1}(\theta)\right| d \theta\right\} d s .
$$


Since $\delta=\mu-2 \gamma L-\varepsilon(v-2 \gamma K)>0$ it follows that

and

$$
I_{1} \leqq 2 K L \gamma\left|w_{1}(\tau)\right| \varepsilon \delta^{-1} e^{-(\nu-2 K \gamma)(t-\tau)}
$$

That is,

$$
I_{2} \leqq 4 K L \gamma^{2} \delta^{-1} \int_{\tau}^{t} e^{-(\nu-2 K \gamma)(t-s)}\left|v_{1}(s)\right| d s
$$

$$
\begin{aligned}
\left|v_{1}(t)\right| \leqq & K e^{-(\nu-2 K \gamma)(t-\tau)}\left(\left|v_{1}(\tau)\right|+2 L \gamma \varepsilon \delta^{-1}\left|w_{1}(\tau)\right|\right) \\
& +4 K L \gamma^{2} \delta^{-1} \int_{\tau}^{t} e^{-(\nu-2 K \gamma)(t-s)}\left|v_{1}(s)\right| d s .
\end{aligned}
$$

By Gronwall's lemma again this inequality gives

$$
\left|v_{1}(t)\right| \leqq K e^{-\lambda(t-\tau)}\left(\left|v_{1}(\tau)\right|+2 L \gamma \varepsilon \delta^{-1}\left|w_{1}(\tau)\right|\right),
$$

where $\lambda=v-2 K \gamma-4 K L \gamma^{2} \delta^{-1}>\frac{1}{2} v$. In a similar manner, we obtain

$$
\left|w_{1}(t)\right| \leqq L e^{-\lambda(t-\tau)}\left(\left|w_{1}(\tau)\right|+2 K \gamma \delta^{-1}\left|v_{1}(\tau)\right|\right) .
$$

Thus for $t \geqq \tau \geqq t_{0}$

$$
\left|v_{1}(t)\right|+\left|w_{1}(t)\right| \leqq(K+L) e^{-\lambda(t-\tau)}\left(\left|v_{1}(\tau)\right|+\left|w_{1}(\tau)\right|,\right.
$$

since $\varepsilon<1$ and $\delta>2 \gamma(K+L)$.

If the solution $v_{i}(t), w_{i}(t), i=1,2$ of (21) does not satisfy

$$
\left|v_{1}(t)\right|+\left|w_{1}(t)\right| \geqq\left|v_{2}(t)\right|+\left|w_{2}(t)\right| \text { for all } t \geqq t_{0},
$$

then we must have

$$
\left|v_{1}\left(t_{1}\right)\right|+\left|w_{1}\left(t_{1}\right)\right|<\left|v_{2}\left(t_{1}\right)\right|+\left|w_{2}\left(t_{1}\right)\right| \text { for some } t_{1} \geqq t_{0} .
$$

Since $\eta=1+(K+L)^{2}>1$ we have throughout some interval $t_{1} \leqq t \leqq t_{2}$,

$$
\left|v_{1}(t)\right|+\left|w_{1}(t)\right| \leqq \eta\left(\left|v_{2}(t)\right|+\left|w_{2}(t)\right|\right) .
$$

From the second and fourth inequalities of (21) it follows respectively that for $t_{1} \leqq \tau \leqq t \leqq t_{2}$,

$$
\begin{aligned}
& \left|v_{2}(\tau)\right| \leqq K e^{-\nu(t-\tau)}\left|v_{2}(t)\right|+K \gamma(1+\eta) \int_{\tau}^{t} e^{-\nu(s-\tau)}\left(\left|v_{2}(s)\right|+\left|w_{2}(s)\right|\right) d s, \\
& \left|w_{2}(\tau)\right| \leqq L e^{-\mu(t-\tau) / \varepsilon}\left|w_{2}(t)\right|+L \gamma \varepsilon^{-1}(1+\eta) \int_{\tau}^{t} e^{-\mu(8-\tau) / \varepsilon}\left(\left|v_{2}(s)\right|+\left|w_{2}(s)\right|\right) d s .
\end{aligned}
$$

Regard $-\tau$ as variable and $-t$ as fixed. Then (23) is of the same form as (22) with $\gamma$ replaced by $\frac{1}{2} \gamma(1+\eta)$, and so by the above argument, we obtain for $t_{1} \leqq \tau \leqq t \leqq t_{2}$

$$
\left|v_{2}(\tau)\right|+\left|w_{2}(\tau)\right| \leqq(K+L) e^{-\lambda_{1}(t-\tau)}\left(\left|v_{2}(t)\right|+\left|w_{2}(t)\right|\right),
$$

since $\gamma, \varepsilon$ have been chosen so small that 


$$
\begin{aligned}
\nu & >K \gamma(1+\eta) ; \quad \mu>L \gamma(1+\eta) \\
\delta_{1} & =\mu-L \gamma(1+\eta)-\varepsilon[\nu-K \gamma(1+\eta)]>0 ; \\
\lambda_{1} & =\nu-K \gamma(1+\eta)-K L \gamma^{2}(1+\eta)^{2} \delta_{1}^{-1}>0 ;
\end{aligned}
$$

and

$$
\varepsilon<1, \quad \delta_{1}>\gamma(K+L)(1+\eta) \text {. }
$$

Now on setting $\tau=t_{1}$ in both the first and third inequalities of (21) we obtain

$$
\begin{aligned}
& \left|v_{1}(t)\right| \leqq K e^{-\nu\left(t-t_{1}\right)}\left|v_{1}\left(t_{1}\right)\right|+K \gamma(1+\eta) \int_{t_{1}}^{t} e^{-\nu(t-s)}\left(\left|v_{2}(s)\right|+\left|w_{2}(s)\right|\right) d s, \\
& \left|w_{1}(t)\right| \leqq L e^{-\mu\left(t-t_{1}\right) / \varepsilon}\left|w_{1}\left(t_{1}\right)\right|+L \gamma \varepsilon^{-1}(1+\eta) \int_{t_{1}}^{t} e^{-\mu(t-s) / \varepsilon}\left(\left|v_{2}(s)\right|+\left|w_{2}(s)\right|\right) d s,
\end{aligned}
$$

and, in view of (24),

$$
\begin{aligned}
\left|v_{1}(t)\right| & \leqq K e^{-\nu\left(t-t_{1}\right)}\left|v_{1}\left(t_{1}\right)\right|+K \gamma(1+\eta)(K+L)\left(\left|v_{2}(t)\right|+\left|w_{2}(t)\right|\right) \int_{t_{1}}^{t} e^{-\left(\nu+\lambda_{1}\right)(t-s)} d s \\
& \leqq K\left|v_{1}\left(t_{1}\right)\right|+K \gamma(1+\eta)(K+L)\left(\nu+\lambda_{1}\right)^{-1}\left(\left|v_{2}(t)\right|+\left|w_{2}(t)\right|\right), \\
\left\{w_{1}(t) \mid\right. & \leqq L\left|w_{1}\left(t_{1}\right)\right|+L \gamma(1+\eta)(K+L)\left(\mu+\varepsilon \lambda_{1}\right)^{-1}\left(\left|v_{2}(t)\right|+\left|w_{2}(t)\right|\right) .
\end{aligned}
$$

Thus it follows that for $t_{1} \leqq t \leqq t_{2}$

$$
\begin{aligned}
\left|v_{1}(t)\right|+\left|w_{1}(t)\right| \leqq & (K+L)\left(\left|v_{2}\left(t_{1}\right)\right|+\left|w_{2}\left(t_{1}\right)\right|\right) \\
& +\gamma(1+\eta)(K+L)^{2}\left[\left(\nu+\lambda_{1}\right)^{-1}+\left(\mu+\varepsilon \lambda_{1}\right)^{-1}\right]\left(\left|v_{2}(t)\right|+\left|w_{2}(t)\right|\right) \\
\leqq & (K+L)^{2}\left\{e^{-\lambda_{1}\left(t-t_{1}\right)}+\gamma(1+\eta)\left[\left(\nu+\lambda_{1}\right)^{-1}+\left(\mu+\varepsilon \lambda_{1}\right)^{-1}\right]\right\} \\
< & \cdot \eta\left(\left|v_{2}(t)\right|+\left|w_{2}(t)\right|\right) .
\end{aligned}
$$

Therefore

$$
\left|v_{1}(t)\right|+\left|w_{1}(t)\right|<\eta\left(\left|v_{2}(t)\right|+\left|w_{2}(t)\right|\right) \text { for all } t \geqq t_{1} .
$$

This completes the proof of lemma B.

\section{5}

In this section we deduce from lemma $B$ the following new results regarding the behaviour as $\varepsilon \rightarrow 0$ of every solution of the system (2) once it enters into the neighbourhood of the unique solution of Theorem 2 .

THEOREM 3. Let the hypotheses of Theorem 2 hold and let $y_{\varepsilon}(t), z_{\varepsilon}(t)$ be the unique solution of Theorem 2. Then for $\varepsilon$ and $\left|y(0)-y_{\varepsilon}(0)\right|+\left|z(0)-z_{\varepsilon}(0)\right|$ sufficiently small any other solution $y(t), z(t)$ of $(2)$ is such that either

(i) $|\tilde{y}(t)|+|\tilde{z}(t)| \leqq C e^{-\lambda(t-\tau)}[|\tilde{y}(\tau)|+|\tilde{z}(\tau)|]$ for $t \geqq \tau \geqq 0$,

or (ii) for some $t_{1} \geqq 0$,

$$
|\tilde{y}(\tau)|+|\tilde{z}(\tau)| \leqq C(1+\eta) e^{-\lambda_{1}(t-\tau)}[|\tilde{y}(t)|+|\tilde{z}(t)|] \text { for } t \geqq \tau \geqq t_{1} \text {, }
$$


where $\tilde{y}(t)=y(t)-y_{\varepsilon}(t), \tilde{z}(t)=z(t)-z_{\varepsilon}(t)$ and $C, \lambda, \lambda_{1}$ and $\eta>1$ are positive constants depending only on $K, L, v$.

Furthermore any solution $y(t), z(t)$ of (2) for which

$$
|y(0)-\bar{y}(0)|+|z(0)-\bar{z}(0)|
$$

is sufficiently small satisfies (i) if and only if it is one of the solutions of Theorem 2 with $\rho$ replaced by $\rho^{\prime}>\rho$.

We shall make use of the following

LEMMA C. Suppose that the linear systems

$$
y^{\prime}=A(t) y, \quad z^{\prime}=B(t) z
$$

have respectively fundamental matrices $Y(t), Z(t)$ such that

$$
\begin{aligned}
\left|Y(t) Y^{-1}(s)\right| & \leqq e^{\alpha|t-s|} & \text { for } & 0 \leqq t, s<\infty, \\
\left|Z(t) \tilde{P} Z^{-1}(s)\right| & \leqq L e^{-\beta(t-s)} & & \text { for } t \geqq s, \\
\left|Z(t)(I-\tilde{P}) Z^{-1}(s)\right| & \leqq L e^{-\beta(s-t)} & & \text { for } s \geqq t,
\end{aligned}
$$

where $\tilde{P}$ is a projection matrix and $L, \alpha, \beta$ are positive constants. If $\alpha<\beta$ then for any continuous bounded matrix function $C(t)$ the system

$$
\begin{aligned}
& y^{\prime}=A(t) y+C(t) z \\
& z^{\prime}=B(t) z
\end{aligned}
$$

is kinematically similar (cf. Markus [11] or Coppel [5]) to the same system (26) with $C(t) \equiv 0$ i.e. to $(25)$.

Proof. Let

$$
\begin{aligned}
T(t)= & \int_{0}^{t} Y(t) Y^{-1}(s) C(s) Z(s)(I-\tilde{P}) Z^{-1}(t) d s \\
& -\int_{t}^{\infty} Y(t) Y^{-1}(s) C(s) Z(s) \tilde{P} Z^{-1}(t) d s .
\end{aligned}
$$

Then

$$
\begin{aligned}
\|T(t)\| & \leqq L\|C(t)\|\left[\int_{0}^{t} e^{(\alpha-\beta)(t-s)} d s+\int_{0}^{\infty} e^{(\alpha-\beta)(s-t)} d s\right] \\
& \leqq 2 L\|C(t)\|(\beta-\alpha)^{-1} .
\end{aligned}
$$

Thus $T(t)$ exists, is continuous and bounded. It is easily verified by differentiation that $T(t)$ is a solution of

$$
T^{\prime}=A(t) T-T B(t)+C(t) .
$$

Consequently the change of variable $y=x+T(t) z$ transforms the first equation of (26) into $x^{\prime}=A(t) x$ and the lemma follows.

Proof of Theorem 3 . We have seen that the change of variables (13) transforms (2) into the system (18). 
Now choose $\sigma>0$ so that $\left|A_{1}(t)\right| \leqq \sigma$ for all $t \geqq 0$. If $\varepsilon<\frac{1}{2} \mu \sigma^{-1}$ then the system

$$
\begin{aligned}
v^{\prime} & =A_{1}(t) v+A_{2}(t) w \\
\varepsilon w^{\prime} & =B(t) w
\end{aligned}
$$

satisfies the conditions of lemma $C$. Thus, by the proof of this lemma, there exists a bounded, continuously differentiable matrix function $T(t)$ such that

and

$$
T^{\prime}=A_{1}(t) T-\varepsilon^{-1} T B(t)+A_{2}(t)
$$

$$
\|T\| \leqq 4 L\left\|A_{2}\right\| \mu^{-1} \varepsilon \text {. }
$$

The change of variable $v=u+T(t) w$ then transforms the nonlinear system (18) into

$$
\begin{aligned}
u^{\prime} & =A_{1}(t) u+\tilde{F}(u, w, t, \varepsilon), \\
\varepsilon w^{\prime} & =B(t) w+\widetilde{G}(u, w, t, \varepsilon),
\end{aligned}
$$

where

$$
\widetilde{F}(u, w, t, \varepsilon)=F(v, w, t, \varepsilon)-\varepsilon^{-1} T(t) G(v, w, t, \varepsilon)
$$

and

$$
\tilde{G}(u, w, t, \varepsilon)=G(v, w, t, \varepsilon)
$$

satisfy conditions of the same type as $F$ and $G$.

Write $\tilde{u}=u(t)-u_{\varepsilon}(t), \tilde{w}=w(t)-w_{\varepsilon}(t)$, where $u(t), w(t)$ is the solution of (27) obtained from $y(t), z(t)$ and $u_{\varepsilon}(t), w_{\varepsilon}(t)$ is the solution obtained from $y_{\varepsilon}(t), z_{\varepsilon}(t)$. Then $\tilde{u}, \tilde{w}$ satisfy

$$
\begin{aligned}
\tilde{u}^{\prime} & =A_{1}(t) \tilde{u}+\tilde{F}(u, w, t, \varepsilon)-\tilde{F}\left(u_{\varepsilon}, w_{\varepsilon}, t, \varepsilon\right), \\
\varepsilon \tilde{w}^{\prime} & =B(t) \tilde{w}+\tilde{G}(u, w, t, \varepsilon)-\tilde{G}\left(u_{\varepsilon}, w_{\varepsilon}, t, \varepsilon\right) .
\end{aligned}
$$

From the variation of constants formula any solution $\tilde{u}(t), \tilde{w}(t)$ of $(28)$ satisfies

$$
\begin{aligned}
\tilde{u}(t)= & V(t) V^{-1}(\tau) \tilde{u}(\tau) \\
& +\int_{\tau}^{t} V(t) V^{-1}(s)\left\{F[u(s), w(s), s, \varepsilon]-F\left[u_{\varepsilon}(s), w_{\varepsilon}(s), s, \varepsilon\right]\right\} d s, \\
\tilde{w}(t)= & W(t) W^{-1}(\tau) \tilde{w}(\tau) \\
& +\varepsilon^{-1} \int_{\tau}^{t} W(t) W^{-1}(s)\left\{\tilde{G}[u(s), w(s), s, \varepsilon]-\tilde{G}\left[u_{\varepsilon}(s), w_{\varepsilon}(s), s, \varepsilon\right]\right\} d s
\end{aligned}
$$

for $t \geqq \tau \geqq 0$. Set

$$
\begin{array}{lll}
\tilde{u}(t)=\tilde{u}_{1}(t)+\tilde{u}_{2}(t), & \text { where } \quad \tilde{u}_{1}(t)=V(t) P V^{-1}(t) \tilde{u}(t), \\
\tilde{w}(t)=\tilde{w}_{1}(t)+\tilde{w}_{2}(t), \quad \text { where } & \tilde{w}_{1}(t)=W(t) P_{1} W^{-1}(t) \tilde{w}(t) .
\end{array}
$$


Then we obtain

$$
\begin{aligned}
\tilde{u}_{1}(t)= & V(t) P V^{-1}(\tau) \tilde{u}_{1}(\tau) \\
& +\int_{\tau}^{t} V(t) P V^{-1}(s)\left\{F[u(s) w(s), s, \varepsilon]-\tilde{F}\left[u_{\varepsilon}(s), w_{\varepsilon}(s), s, \varepsilon\right]\right\} d s, \\
\tilde{u}_{2}(\tau)= & V(\tau)\left(I_{m}-P\right) V^{-1}(t) \tilde{u}_{2}(t) \\
& -\int_{\tau}^{t} V(\tau)\left(I_{m}-P\right) V^{-1}(s)\left\{F[u(s), w(s), s, \varepsilon]-\tilde{F}\left[u_{\varepsilon}(s), w\left(s_{\varepsilon}\right), s, \varepsilon\right]\right\} d s, \\
\tilde{w}_{1}(t)= & W(t) P_{1} W^{-1}(\tau) \tilde{w}_{1}(\tau) \\
& +\varepsilon^{-1} \int_{\tau}^{t} W(t) P_{1} W^{-1}(s)\left\{\tilde{G}[u(s), w(s), s, \varepsilon]-\tilde{G}\left[u_{\varepsilon}(s), w_{\varepsilon}(s), s, \varepsilon\right]\right\} d s, \\
\tilde{w}_{2}(\tau)= & W(\tau)\left(I_{n}-P_{1}\right) W^{-1}(t) \tilde{w}_{2}(t) \\
& -\varepsilon^{-1} \int_{\tau}^{t} W(\tau)\left(I_{n}-P_{1}\right) W^{-1}(s) \\
& \cdot\left\{\tilde{G}[u(s), w(s), s, \varepsilon]-\tilde{G}\left[u_{\varepsilon}(s), w_{\varepsilon}(s), s, \varepsilon\right]\right\} d s .
\end{aligned}
$$

It follows from (10), (12) that for $t$ in some interval $\tilde{\tau} \geqq t \geqq \tau \geqq 0$,

$$
\begin{aligned}
\left|\tilde{u}_{1}(t)\right| & \leqq K e^{-\nu(t-\tau)}\left|\tilde{u}_{1}(\tau)\right|+K \delta \int_{\tau}^{t} e^{-\nu(t-s)}(|\tilde{u}(s)|+|\tilde{w}(s)|) d s, \\
\left|\tilde{u}_{2}(\tau)\right| & \leqq K e^{-\nu(t-\tau)}\left|\tilde{u}_{2}(t)\right|+K \delta \int_{\tau}^{t} e^{-\nu(s-\tau)}(|\tilde{u}(s)|+|\tilde{w}(s)|) d s, \\
\left|\tilde{w}_{1}(t)\right| & \leqq L e^{-\mu(t-\tau) / \varepsilon}\left|\tilde{w}_{1}(\tau)\right|+L \delta \varepsilon^{-1 / \varepsilon} \int_{\tau}^{t} e^{-\mu(t-s) / \varepsilon}(|\tilde{u}(s)|+|\tilde{w}(s)|) d s, \\
\left|w_{2}(\tau)\right| & \leqq L e^{-\mu(t-\tau) / \varepsilon}\left|\tilde{w}_{2}(t)\right|+L \delta \varepsilon^{-1 / \varepsilon} \int_{\tau}^{t} e^{-\mu(s-\tau) / \varepsilon}(|\tilde{u}(s)|+|\tilde{w}(s)|) d s .
\end{aligned}
$$

The functions $\tilde{u}_{1}(t), \tilde{u}_{2}(t), \tilde{w}_{1}(t), \tilde{w}_{2}(t)$ satisfy all the conditions of lemma B so that if $\delta, \varepsilon$ are sufficiently small, we obtain either case (i)

$$
\begin{aligned}
|\tilde{u}(t)|+|\tilde{w}(t)| & \leqq 2\left(\left|\tilde{u}_{1}(t)\right|+\left|\tilde{w}_{1}(t)\right|\right) & & \text { for } t \geqq \tau \geqq 0 \\
& \leqq 2(K+L) e^{-\lambda(t-\tau)}\left(\left|\tilde{u}_{1}(\tau)\right|+\left|\tilde{w}_{1}(\tau)\right|\right) & & \text { for } t \geqq \tau \geqq 0,
\end{aligned}
$$

or case (ii), for some $t_{\mathbf{1}} \geqq 0$,

$$
\begin{aligned}
|\tilde{u}(\tau)|+|\tilde{w}(\tau)| & \leqq(1+\eta)\left(\left|\tilde{u}_{2}(\tau)\right|+\left|\tilde{w}_{2}(\tau)\right|\right) & & \text { for } \quad \tau \geqq t_{1}, \\
& \leqq(1+\eta)(K+L) e^{-\lambda_{1}(t-\tau)}\left(\left|\tilde{u}_{2}(t)\right|+\left|\tilde{w}_{2}(t)\right|\right) & & \text { for } \quad t \geqq \tau \geqq t_{1} .
\end{aligned}
$$

Since, in addition,

$$
\begin{aligned}
\left|\tilde{u}_{1}(t)\right|,\left|\tilde{u}_{2}(t)\right| & \leqq K|\tilde{u}(t)|, \\
\left|\tilde{w}_{1}(t)\right|,\left|\tilde{w}_{2}(t)\right| & \leqq L|\tilde{w}(t)|,
\end{aligned}
$$

we obtain case (i) and case (ii) of Theorem 3 .

We now prove the last statement of Theorem 3. If $y(t), z(t)$ is one of the solutions of Theorem 2, then it follows that it is bounded for all $t \geqq 0$ and therefore cannot satisfy (ii), for otherwise it becomes unbounded for $t$ large 
enough. Hence the solution must satisfy (i). Conversely, let $y(t), z(t)$ be a solution of (2) which satisfies (i) and for which

$$
d=|y(0)-\bar{y}(0)|+|z(0)-\bar{z}(0)|
$$

is sufficiently small. Choose $d$ such that $C d \leqq \rho^{\prime}-\rho$ and

$$
d\left[|P|+\left|P_{1}\right|\{1+|U(0)|\}\right] \leqq \gamma
$$

where $\rho, \gamma$ are as in Theorem 2. Then

$$
\begin{aligned}
|P[y(0)-\bar{y}(0)]|+\mid P_{1}[z(0)-\bar{z}(0) & +U(0)\{y(0)-\bar{y}(0)\}] \mid \\
& \leqq d\left[|P|+\left|P_{1}\right|\{1+|U(0)|\}\right] \leqq \gamma
\end{aligned}
$$

and by (i)

$$
|y(t)-\bar{y}(t)|+|z(t)-\bar{z}(t)| \leqq C d+\left|y_{\varepsilon}(t)-\bar{y}(t)\right|+\left|z_{\varepsilon}(t)-\bar{z}(t)\right| \leqq \rho^{\prime} .
$$

It follows that $y(t), z(t)$ is indeed one of the solutions of Theorem 2 with $\rho$ replaced by $\rho^{\prime}$. This completes the proof of Theorem 3 .

\section{6}

In this last section we obtain by an application of Theorem 3 the following theorem which gives a slightly stronger result than Anosov's Theorem $5[1]$.

THEOREM 4. Assume, in addition to the assumptions (I)-(IV) of Theorem 1 , that $(\mathrm{V})$ the variational equation (4) admits a single multiplier equal to 1 in modulus. Let $y_{\varepsilon}(t), z_{\varepsilon}(t)$ be the periodic solution of Theorem 1. Then for $\varepsilon$ and $\left|y(0)-y_{\varepsilon}(0)\right|+\left|z(0)-z_{\varepsilon}(0)\right|$ sufficiently small any solution $y(t), z(t)$ of (1) is such that either

(i) $|\tilde{y}(t)|+|\tilde{z}(t)| \leqq C e^{-\lambda(t-\tau)}[|\tilde{y}(\tau)|+|\tilde{z}(\tau)|]$ for $t \geqq \tau \geqq 0$, or (ii) for some $t_{1} \geqq 0$,

$$
|\tilde{y}(\tau)|+|\tilde{z}(\tau)| \leqq C_{1} e^{-\lambda_{1}(t-\tau)}[|\tilde{y}(t)|+|\tilde{z}(t)|] \text { for } t \geqq \tau \geqq t_{1},
$$

where $\tilde{y}(t)=y(t)-y_{\varepsilon}(t), \tilde{z}(t)=z(t)-z_{\varepsilon}(t)$ and $C, C_{1}, \lambda, \lambda_{1}$ are some positive constants.

Proof. By the change of variables (5) we have transformed (1) into (6) which is of the same form as (2) with $\theta$ in place of $t$. The additional assumption $(\mathrm{V})$ of the theorem implies that the linear equation $d v / d \theta=A_{1}(\theta) v=C(\theta) v$ has a fundamental matrix $V(t)$ such that (10) holds. It is not difficult to verify that (6) in fact satisfies all the hypotheses of Theorem 3 so that Theorem 4 follows as a consequence of Theorem 3.

The author is greatly indebted to $\mathrm{Mr} \mathrm{W}$. A. Coppel for his guidance and interest in this work. 


\section{References}

[1] D. V. Anosov, 'Limit cycles of systems of differential equations with small parameters in the highest derivatives', Mat. Sb. (N.S.) 50 (92) (1960), 299-334; Amer. Math. Soc. Transl. (2) 33 (1963), 233-276.

[2] V. F. Butuzov, 'Asymptotic behaviour of solutions of differential equations with a small parameter in the derivative on a semi-infinite interval', Vestnik Moskov. Univ. Ser. I Mat. Meh. no. 1 (1965), 16-25.

[3] K. W. Chang, 'Almost periodic solutions of singularly perturbed systems of differential equations', J. Diff. Eq. 4 (1968). 300-307.

[4] W. A. Coppel, 'Stability and asymptotic behaviour of differential equations', (D.C. Heath, Boston 1965).

[5] W. A. Coppel, 'Dichotomies and reducibility', J. Diff. Eq. 3 (1967), 500-521.

[6] K. O. Friedrichs and W. R. Wasow, 'Singular perturbations of nonlinear oscillations'. Duke Math. J. 13 (1946), 367-381.

[7] A. Halanay, Differential equations: stability, oscillations, time lags, (Academic Press, New York, 1966).

[8] F. C. Hoppensteadt, 'Singular perturbations on the infinite interval', Trans. Amer. Math. Soc. 123 (1966), 521-535.

[9] F. C. Hoppensteadt, 'Stability in systems with parameter', J. Math. Anal. Appl. 18 (1967), 129-134.

[10] J. J. Levin, 'Singular perturbations of nonlinear systems of differential equations related to conditional stability', Duke Math. $J .23$ (1956), 609-620.

[11] L. Markus, 'Continuous matrices and the stability of differential systems', Math. $Z$. 62 (1955), $310-319$.

[12] A. N. Tihonov, 'Systems of differential equations containing a small parameter multiplying the derivative', Mat. Sb. 31 (73) (1952), 575-586.

[13] M. Urabe, 'Geometric study of nonlinear autonomous system', Funkcial. Ekvaj. 1 (1958), $1-84$.

Department of Mathematics

Institute of Advanced Studies

Australian National University

Canberra

and

University of Calgary 